\title{
Proteomics: An Indispensable Tool for Novel Biomarker Identification in Melanoma
}

Payal Mittal ${ }^{1 *}$ and Manish Jain²

${ }^{1}$ Department of Immunology, University of Connecticut Health Center, Farmington, CT 06030, USA

${ }^{2}$ Center for Addiction Research, University of Texas Medical branch, Galveston, TX 77555, USA

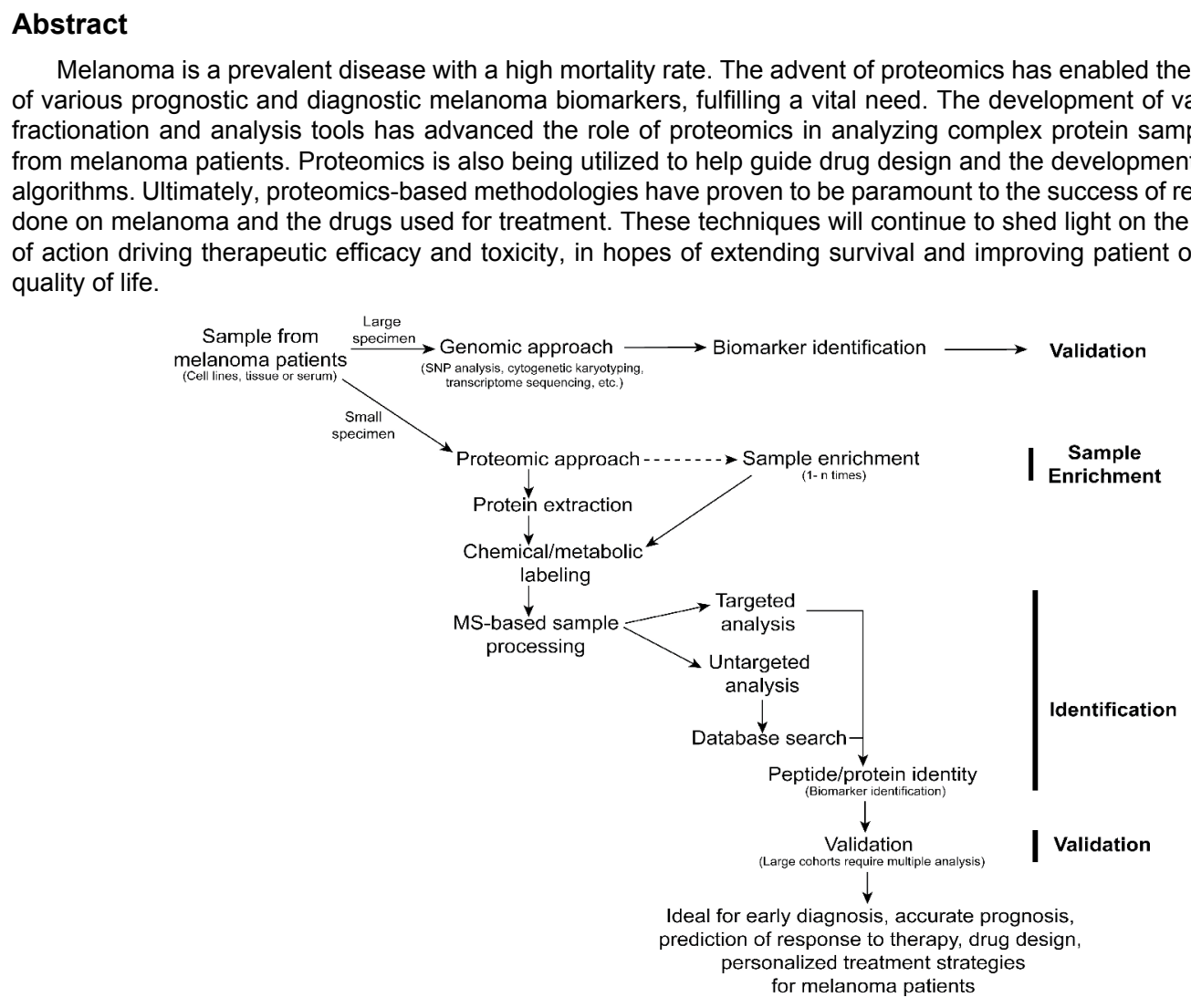

Keywords: Melanoma; Biomarkers; Tumor; Prognosis; Diagnosis; Proteomics; LDH

\section{Introduction}

Skin cancers are the most common cancer worldwide. Among the many types of skin cancers, melanoma is the most deadly. It is the $5^{\text {th }}$ and $6^{\text {th }}$ most prevalent cancer in males and females respectively and is predicted by American Cancer Society to account for approximately 76,380 cases in the United States this year alone. Common risk factors associated with melanoma include excessive exposure to UV radiation, coal, arsenic compounds and immune suppression [1]. The high rate of metastases associated with melanoma limits prognosis as well as makes diagnosis and treatment of melanoma extremely difficult [2]. Treatment strategies also struggle to take into account the high frequency of mutations in response to environmental stress or therapeutic pressures leading to inter-tumoral and/or intra-tumoral heterogeneity [3]. Successful treatment of melanoma depends critically on overcoming these obstacles.

Early stage melanomas are removed surgically. For more advanced or metastatic melanomas, however, we must turn towards more sophisticated therapeutic approaches. In the past, chemotherapy was the default, standard-of-care treatment option. Due to their superior efficacy, immunomodulatory antibodies are now replacing previous therapies as standard-of-care. Immunomodulatory antibodies are broadly comprised of immunostimulatory and immunoinhibitory antibodies. Combinations of immunotherapeutics, as well as in combination with traditional chemotherapy or radiation therapy, are yielding even greater efficacy in patients. Among immunostimulatory antibodies used for targeted therapies, CD28, CD40, CD134 (OX40) and $\mathrm{CD} 137(41 \mathrm{BB})$ are fairly common. A targeted combinatorial

*Corresponding author: Payal Mittal, Department of Immunology, University of Connecticut Health Center, Farmington, CT 06030, USA, Tel: 860-920-9297; E-mail: pmittal@uchc.edu

Received July 05, 2016; Accepted July 18, 2016; Published July 25, 2016

Citation: Mittal P, Jain M (2016) Proteomics: An Indispensable Tool for Novel Biomarker Identification in Melanoma. J Data Mining Genomics Proteomics 7: 204. doi:10.4172/2153-0602.1000204

Copyright: $\odot 2016$ Mittal P, et al. This is an open-access article distributed unde the terms of the Creative Commons Attribution License, which permits unrestricted use, distribution, and reproduction in any medium, provided the original author and source are credited. 
immunotherapy using agonistic monoclonal antibodies CD134 and CD137 has been shown to be effective against B16F10 melanoma in a therapeutic model in mice [4]. Among co-inhibitory antibodies ipilimumab (anti-cytotoxic T-lymphocyte-associated protein 4; antiCTLA-4), nivolumab and pembrolizumab (anti-programmed cell death protein 1; anti-PD-1) have been shown to have superior efficacy against melanoma. Combination therapy including ipilimumab and GMCSF (granulocyte macrophage colony-stimulating factor) has been shown to be superior in terms of efficacy and safety in comparison to ipilimumab monotherapy during a phase II clinical trial in patients with metastatic melanoma [5]. Similarly, in a phase III trial, ipilimumab and nivolumab in combination demonstrated a favorable safety profile and higher therapeutic efficacy than nivolumab or ipilimumab alone against advanced melanomas [6]. Keeping in view the many hurdles associated with melanoma treatment, it is apparent that we need to define better indicators of various stages of disease, to allow for earlier diagnosis, more accurate prognosis, and greater precision in designing patient-tailored therapeutic regimens.

This review discusses the use of proteomic tool in identifying these biological indicators termed as biomarkers and discusses its advantages compared to genomic approaches. Various proteomic platforms are considered in the context of melanoma biomarker identification and characterization for both primary and metastatic disease. We also described limitations and disadvantages associated with proteomic approaches along with potential strategies to overcome them.

\section{Genomic Approach to Biomarker Identification}

Biomarkers are attractive clinical tools that facilitate early detection of disease, as well as its state of progression or regression and even likelihood of response to a particular clinical intervention [7]. Generally speaking, biomarkers identify the alterations of body fluid constituents or tissue composition corresponding to a disease state. Biological fluids such as blood, urine and other body fluids like cerebrospinal fluids are generally utilized as source of biomarkers. Biomarkers used in cancer studies can be predictive biomarkers. These may predict for example, response to a specific therapeutic intervention during disease progression. Somatic mutations in a GTPase encoding KRAS (V-Kiras2 Kirsten rat sarcoma viral oncogene homolog) gene is a standard predictive biomarker in determining eligibility of patients with advanced colorectal carcinoma for targeted therapy with cetuximab or panitumumab directed against epidermal growth factor receptor (EGFR) [8]. Likewise, missense somatic mutation in BRAF (v-Raf murine sarcoma viral oncogene homolog $B)$ gene $(\mathrm{V} 600 \mathrm{E} / \mathrm{K} / \mathrm{D} / \mathrm{R} / \mathrm{M})$ is a predictive biomarker in patients with advanced melanoma [9]. Biomarkers that enable the assessment of cancer progression, tumor stage and malignancy status, likely disease course, and probability of remission are referred to as prognostic biomarkers. These are helpful in providing information regarding clinical outcome such as cancer recurrence or its progression in future. Mutations within the genes encoding glutathione S-transferases [glutathione S-transferase mu 1 (GSTM1), glutathione S-transferase theta 1 (GSTT1), glutathione S-transferase pi 1 (GSTP1)] and a polymorphism within the gene encoding prostate-specific antigen (PSA) are both predictive biomarkers indicating increased risk of prostate cancer [10,11]. Until recently, no biomarker was available for pembrolizumab treatment in melanoma patients. Weide et al., reported a prognostic model for patients treated with pembrolizumab involving four baseline factors: Serum lactate dehydrogenase (LDH) levels, relative lymphocyte count, relative eosinophil count and visceral metastasis patterns [12]. However, this model needs to be validated in randomized controlled trials of pembrolizumab to determine prognostic benefit value in guiding treatment decisions. Additionally, diagnostic markers are used to identify specific disease conditions in individual patients. Three serum microRNAs, miR-720, miR-1308 and miR-1246, were found to be potential diagnostic biomarkers in myeloma patients [13]. Increases in lymphocyte and eosinophil counts in response to ipilimumab have been found to be associated with improved survival in metastatic melanoma patients [14].

Several labs are using genomic approaches to identify biomarkers at the gene level and to define distinct molecular phenotypes associated with different stages of melanoma progression. In this context, gene expression signatures associated with melanoma progression from nevus to primary melanoma to metastatic melanoma, have been identified using microarray studies [15]. Also, uveal melanoma genomic signatures have been profiled via karyotype analysis, fluorescence in situ hybridization (FISH) and comparative genomic hybridization [16]

Genomic data is helpful in analyzing tumors with high resolution. Areas of study include genome-wide DNA methylation patterns, gene expression, single nucleotide polymorphisms (SNPs) and copy number variations. Such approaches can be applied to both primary melanoma tumors and to metastases to lymph nodes, brain and to other organs [17].

Despite the usefulness of genome profiling for molecular biomarkers identification, there are several shortcomings associated with this approach. The requirement for fresh specimens from large numbers of patients may be prohibitive. In most cases of melanoma, primary tumors are small and may not provide adequate tissue amounts. Another obstacle to genomic approaches is that the biomarkers are identified at the gene level only. However, functionality depends on the hierarchical transition from gene to protein within each cell (Figure 1). RNAs resulting from transcription may exist as several splice variants resulting from alternate splicing, leading to different isoforms of the same protein. Changes in any of these protein isoforms could potentially serve as prognostic biomarkers for melanoma, but these could be missed by genomic approaches. Additionally, proteins often undergo post-translational modifications (PTMs) [18]. Aberrant protein structure due to defects in PTMs, environmental or chemical stress, protein degradation and defect in protein-protein interactions could serve as hallmarks of tumor progression. However, these factors can be overlooked by genomic profiling approaches. Despite these shortcomings, genomics has some promise in understanding the molecular basis of melanoma.

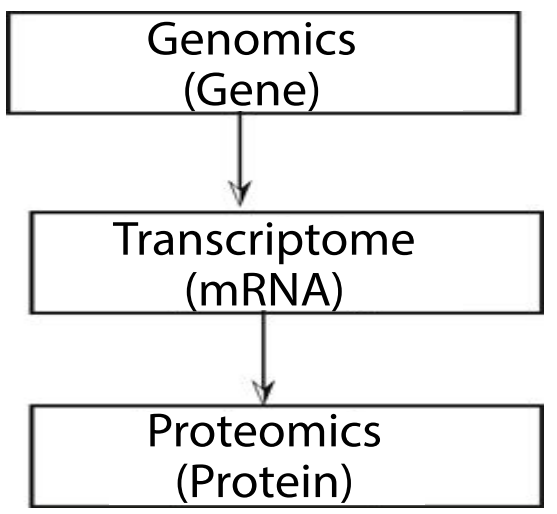

Figure 1: The hierarchical transition from gene to protein within each cell. 


\section{Utilizing the Power of the Proteome}

Proteome analysis aims to uncover dynamics between genes and environment and hence is uniquely poised for efficient biomarker discovery. Under any disease conditions, proteins are most likely to be affected and hence, can serve as excellent biomarker substrates. A schematic of a representative proteome workflow is shown in Figure 2. Proteome studies have successfully identified biomarkers associated with aberrant protein expression in tumor conditions $[19,20]$. With new peptide/protein separation technologies, isotope labeling for identification, and bioinformatic data analysis tools, proteomic studies possess with higher accuracy, sensitivity and resolution. Characterization of a protein by analyzing peptides generated by proteolytic digestion is termed bottom-up proteome analysis. Utilizing bottom-up analysis for analyzing a complex mixture of proteins is termed shotgun proteomics [21]. This approach is widely used for biomarker discovery associated with different disease conditions due to its very high sensitivity and precision. However, realistically it has got lot of limitations associated with it. Peptic digestion results into generation of complex mixture of peptides, making high-throughput screening difficult. Proteolytic digestion, can miss various proteins that are not properly denatured, can result into peptide fragments that are too short for detection, can skip a cleavable site or can miss PTM resulting into inaccurate protein quantification [22]. Another problem associated with this approach is underrepresentation of membrane proteins due to their poor solubility [23]. This resulted into decrease in depth and accuracy of proteome analysis. Alternatively, top-down proteomics involves characterization of intact proteins. It has potential advantages in PTMs and isoform identification, however disadvantages include difficulties with protein fractionation, ionization and fragmentation. Shotgun proteomics is widely used for biomarker identification in numerous cancer types.

\section{Enzyme-linked immunosorbent assay (ELISA) and immunohistochemistry (IHC)}

ELISA is most common diagnostic tool and biochemistry assay that involves the utilization of an antibody specific to a particular antigen. The antigen specific antibody in turn is linked to an enzyme. Upon addition of substrate for the enzyme, the reaction produces a detectable signal, which is mostly a color change in substrate and is directly

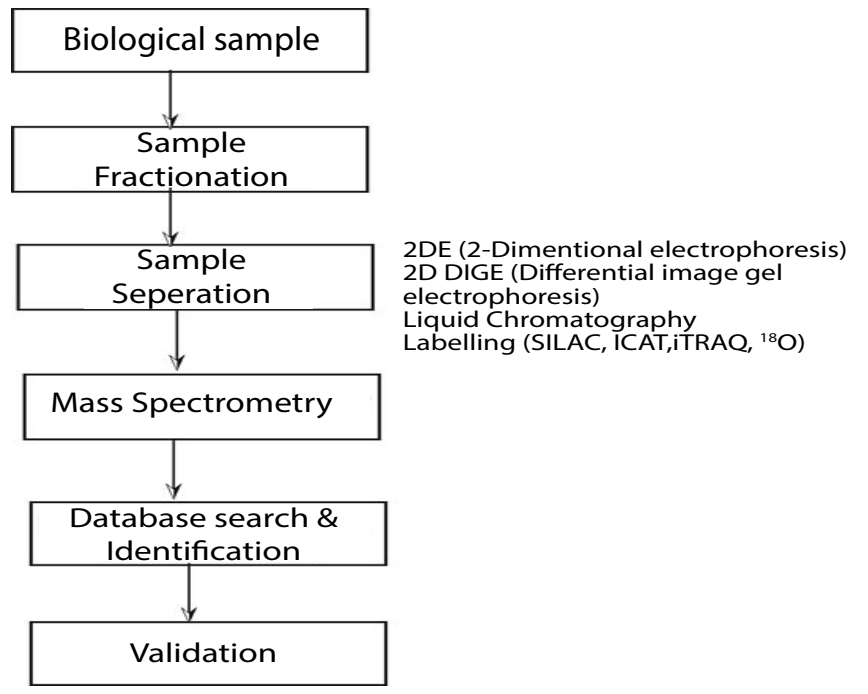

Figure 2: A schematic of a representative proteome workflow. proportional to levels of antigen present in the sample. ELISA is most reliable and sensitive method for protein quantification for the purpose of clinical diagnostics. Immunohistochemistry (IHC) is another antigen specific antibody based technique to detect antigens of interest in cells of a tissue. It is capable of localizing proteins within a cell or tissue however; it is unable to quantitate protein amounts and is more of a visualization technique. Neither of the above mentioned techniques are suitable for target discoveries due to low throughput requirements. Mass spectrometry (MS) is a comparatively newer technique that overcomes both of these limitations.

\section{Mass spectrometric (MS) analysis}

It combines protein identification with quantitative measurements and is very useful in the development of new technologies. Trypsin digested peptides, derived from different biological samples (e.g., tissue or plasma-derived proteins) can be analyzed using different modes of MS. Untargeted modes of MS can discover novel protein biomarker candidates from tissues and biological fluids. A study conducted on stage IV melanoma patients, utilized untargeted MS mode to identify the association of alpha 1-acid glycoprotein precursor-1/2 (AAG-1/2) with metastasis [24]. In contrast, a targeted mode enables to identify peptide of interest in clinical samples. A version of targeted MS was specifically developed to detect $\mathrm{ng} / \mathrm{ml}$ range of plasma concentrations of pigment intermediate 5-S-cysteinyldopain which is generally utilized for early diagnosis, evaluation of treatment as well as malignant melanoma progression [25]. MS is a great tool to analyze complex protein samples. It has significantly augmented the reliability, reproducibility and efficiency of proteomic studies. MS analysis typically comprises of an ionization source, a mass analyzer and a detector. Various ionization techniques such as electrospray ionization (ESI), matrix-assisted laser desorption/ionization (MALDI) are commonly used techniques [2628]. Peptides are introduced to an ionization source where they are ionized to gas phased charged particles and which are than separated by a mass analyzer using magnetic fields based on their mass-tocharge ratios $(\mathrm{m} / \mathrm{z})$. The beam of ions passing through the machine is detected; signal is amplified and recorded as a mass spectrum. Mass determination accuracy and resolution is further enhanced with a combined quadrupole-time of flight (Q-TOF) mass analyzer. MS is able to identify peptide fragments with high accuracy and specificity. Widely used peptide fragmentation method are collision induced dissociation (CID) and electron transfer dissociation (ETD) [29]. After obtaining raw data, protein identification and quantification is achieved by searching available databases utilizing various bio-analytical softwares.

\section{Gel based proteomic analysis}

2D-PAGE is the most conventional method of separating complex protein mixtures. Proteins are subjected to two-dimensional gel electrophoresis, which separates in one direction based on their isoelectric point $(\mathrm{pI})$ and in another direction based on their mass. Following 2D-PAGE separation, proteins can be identified using MALDI-MS or LC-MS. Gels are stained in order to visualize different protein spots and are analyzed using 2D gel analysis software (14). Increases in transthyretin (TIR) and angiotensinogen (AGT) with decrease in expression of vitamin D binding protein (DBP) was identified as potential biomarkers in cutaneous malignant melanoma utilizing serum proteome map generated by 2D-PAGE [30]. Release of alpha-N-acetylgalactosaminidase enzyme by cancer cells deglycosylates DBP and hence interferes with DBP mediated immune cascade leading to immunosuppression in melanoma patients. Enzymatic activity of alpha-N-acetylgalactosaminidase enzyme was significantly increased in stage III melanoma patients as compared to early stages suggesting 
that assessing the enzymatic activity of this enzyme may serve as a non-invasive way of evaluating melanoma severity. Additionally, a hypoxia-inducible promoter-adhE screened from hypoxia-regulated endogenous proteins of Salmonella using 2D-PAGE has been used to investigate anticancer efficacy of attenuated Salmonella typhimurium VNP20009 (VNP) to deliver human endostatin in murine melanomas and Lewis lung carcinoma models [31]. 2D-PAGE has also been used to analyze protein lysates of A375 human malignant melanoma cells with or without arbutin treatment and identified upregulation of 14-33 protein gamma (14-3-3g), voltage-dependent anion-selective channel protein 1 (VDAC-1) and tumor suppressor p53 and downregulation of endoplasmin precursor (EMPL), alpha-enolase (ENOA), inosine-5' monophosphate dehydrogenase 2 (IMDH2), peroxiredoxin-1 (PRDX1) and vimentin (VIME) as potential biomarkers for suppression of cancer development [32]. Recently, protein separation with 2D-PAGE and expression analysis between primary melanoma and lymph node metastasis, melanoma differentiation associated gene-9 (MDA-9) and $78 \mathrm{kDa}$ glucose-regulated protein (GRP78) have been identified as potential diagnostic biomarkers for detection of early metastatic melanoma [33]. However, a major shortcoming of 2D-PAGE is variability and reproducibility. To increase reliability and sensitivity, Alban et al., developed two dimensional difference gel electrophoresis (2-D DIGE) [34]. This method involves the differential fluorescent labeling of samples from diseased patients and healthy controls and simultaneous resolution on the same gel. Resultant spots are compared to a master gel of a pool of both samples, and differentially expressed proteins are determined and processed for protein identification. Linge et al., identified 14 differentially expressed proteins (PDIA3, VIM/ HEXA, SELENBP1, ENO1, CAPZA1, ERP29, TP11, PARK7 and FABP3, EIF2S, PSMA3, RPSA, TUBB and TUBA1B) using 2-D DIGE between uveal melanoma patients who subsequently did or did not developed metastases [35]. Likewise, differential protein expression profiles of parental mouse melanoma B16F10 and corresponding lung metastasis by employing 2-D DIGE identified vimentin as potential biomarker for predicting melanoma hematogenous metastasis [36].

Following gel separation, protein spots can be subjected to trypsin digestion and analyzed by MALDI-ToF, yielding peptide mass fingerprint. Alternatively, liquid chromatography tandem-mass spectrometry (LC-MS/MS), can generate information regarding peptide sequence. Using human primary uveal melanoma tumors, molecular differences in metastatic verses non-metastatic tumors were detected by employing LC-MS/MS technology and this resulted into identification of collagen alpha-3(VI) and heat shock protein beta-1 as candidate biomarkers for uveal melanoma metastasis [37]. Proteomic analysis of melanoma cell lines and human melanocytes using 2D-PAGE and LC-MS/MS revealed 6 potential biomarkers (galectin-1, inosine-5'-monophosphate dehydrogenase 2 , serine/threonine-protein phosphatase $2 \mathrm{~A} 65 \mathrm{kDa}$ regulatory subunit $\mathrm{A} a$ isoform, protein $\mathrm{DJ}$ 1 , cyclophilin A and cofilin-1) that were up regulated in melanoma cell lines compared to melanocytes [38]. Additionally, a LC-MS-based label-free method was utilized to identify changes in protein expression levels of 120 proteins associated with melanoma metastasis compared with primary melanomas [39]. Other mass spectrometric techniques such as ESI-MS/MS can provide amino acid sequences of peptide fragment from parent protein. One of the studies validated SPEC-LCESI-MS/MS for determination of 5-S-cysteinyldopa, a human plasma melanoma biomarker [40].

\section{Label based proteomics}

Labeling of proteins is helpful in quantification studies. Labeling techniques can involve either chemical or metabolic labeling.
Chemical labeling is the simplest form of labeling. It involves proteolytic digestion, which is performed in presence of heavy water $\left(\mathrm{H}_{2}^{18} \mathrm{O}\right)$. Serine proteases can than incorporate heavy ${ }^{18} \mathrm{O}$ upon peptidic amide bond cleavage. This is not used very often due to variability in exchange reaction efficiency amongst different peptides [41]. Quantitative analysis of plasma membrane proteins from hypoxia-adapted B16F10 murine melanoma using differential ${ }^{16} \mathrm{O} /{ }^{18} \mathrm{O}$ isotopic labeling and LC-MS, identified consistent increases in transcriptomic and proteomic expression of aminopeptidase $\mathrm{N}$ (CD13), carbonic anhydrase IX, potassium-transporting ATPase, matrix metalloproteinase 9, and stromal cell derived factor-I (SDF1). Antibody-based analysis of a panel of human melanoma cell lines subsequently confirmed consistent upregulation of CD13 and SDF-1 during hypoxia [42].

Isotope-coded affinity tags (ICAT) represent another labeling technique to identify cysteine-containing proteins. It involves a cysteine sulfhydryl reactive chemical group, an isotopically-coded linker and a biotin tag for affinity purification. Cysteine-containing proteins are isotopically labeled, pooled, proteolytically cleaved and subjected to affinity purification using biotin-avidin affinity columns prior to MS analysis. A weakness of this method, however, is that it can only be applied to cysteine-containing proteins and lot of proteins lacks this amino acid [43]. Differential profiling of breast cancers using the ICAT labeling approach followed by tandem mass spectrometry enabled identification of biotinidase as a breast cancer marker [44].

Another labeling approach known as Isotope coded protein label (ICPL) is based on isotope labeling of all accessible amino acids present within protein mixtures using different derivatives of deuterium under reducing and alkylating conditions $[45,46]$. Coupling ICPL with insolution isoelectric fractionation and LC-MALDI-TOF/TOF has been used to validate increases of HE4 and osteopontin as well as identification of phosphatidylethanolamine binding protein 1 and cell adhesion molecule 1 as potential ovarian cancer biomarkers in urine samples [47].

Peptides can also be labeled with isobaric tags for relative and absolute quantification (iTRAQ), which labels peptide N-termini and e-amino group lysine side chains [48]. It can analyze up to four protein samples simultaneously. Potential prognostic markers for stage III melanoma were identified using iTRAQ in conjunction with 2DLCMS/MS to comprehensively profile lymph node metastases in stage III patients with either good or poor survival [49]. iTRAQ was also used to perform quantitative proteomic comparisons of patients with or without metastatic uveal melanoma. This resulted in identification of collagen alpha-3(VI) and heat shock protein beta-1 as candidate biomarkers [37].

Tandem mass tag (TMT) is another MS/MS-based quantitative method [50]. This technique involves cysteine-reactive reagents that can selectively determine relative quantities of cysteine-containing peptides from up to 6 specimens. Salivary proteome analysis using a TMT approach to compare cancer patients to healthy controls was able to identify biomarker signatures associated with gastric cancer [51].

In principle, combination of these techniques could be utilized for the identification of novel melanoma biomarkers.

\section{Metabolic labeling}

Metabolic labeling methods are becoming more widely used in melanoma proteomic studies [52]. Stable isotopic labeling with amino acids in cell culture (SILAC) is one of the more common in vivo labeling techniques. Cells are cultured in media with amino acids containing 
isotopes such as ${ }^{15} \mathrm{~N}$ or ${ }^{13} \mathrm{C}$, which gets taken up by the cells. It enables to introduce fixed mass shift between labeled and unlabeled peptide pairs by labeling of only specific amino acids such as lysine, arginine or leucine. MS can then be used to analyze mass differences between heavy and light cells [53]. Using this strategy, differential plasma membrane protein expression has been shown to correlate with melanoma metastatic potential. This study further identified CUB domaincontaining protein 1 (CDCP1) as one of the surface markers involved in tumor metastasis [54].

In addition to SILAC, ${ }^{14} \mathrm{~N} /{ }^{15} \mathrm{~N}$ labeling is another metabolic labeling technique. Using this approach, whole cells can be labeled in growth media. Label incorporation rates with ${ }^{14} \mathrm{~N} /{ }^{15} \mathrm{~N}$ are higher than with SILAC. However, with this method all amino acids get labeled. Therefore, the overall mass shift is protein sequence dependent [55].

\section{Peptide fractionation techniques}

A key for successful proteomic study is to increase proteome coverage including low abundant proteins without modifying protein/peptide samples. Protein fractionation enables the resolution of the peptides either through one separation system or using a multidimensional approach in order to increase proteome coverage further.

Ion exchange chromatography (IEC): It is a technique with high resolution and capacity. It performs peptide separation according to electric charge. Cation-exchange chromatography (CX) relies on the attraction of positively charged peptides to negative functional group at low $\mathrm{pH}$, whereas anion-exchange chromatography (AX) relies on the attraction of negatively charged peptides to positive functional groups at high $\mathrm{pH}$. Peptides are initially separated according to charge, and then resolved according to isoelectric point ( $\mathrm{pI}$ ) using a $\mathrm{pH}$ gradient. Utilizing this fractionation method along with deep protein sequencing, novel biomarkers associated with metastatic melanoma could be identified from lymph node metastases [56].

Reverse phase chromatography (RP-LC) can be used to separate neutral peptides according to their hydrophobicity. This widely used liquid chromatographic method is dependent on partition coefficients of the analyte between polar mobile phase and the non-polar stationary phase. Various diagnostic and prognostic biomarkers have been identified with RP-LC-based serum profiling of breast cancer patients and healthy controls [57].

Another chromatographic technique known as two-dimensional liquid chromatography (2D-LC). It involves orthogonal combination of two individual chromatographic techniques. The purpose of this multi-dimensional approach is to compensate for sample complexity and improve resolution with maximum proteome coverage, For example coupling of AX to RP, CX to RP or affinity chromatography to RP and so on.

\section{CyTOF}

Analysis of intact proteins is relevant to translational studies. This involves the areas related to bioassays that might advance biomarker discovery related to melanoma progression and associated signaling cascades. Immunological assays for testing various protein modifications that takes place during disease progression for example, phosphorylation of proteins, active proteases, protein ubiquitination, nitration and several other protein modifications might be helpful in novel biomarker discoveries. Single-cell mass cytometry or CyTOF is a MS based flow cytometry technique. It utilizes antibodies tagged with stable metal isotopes for cell staining, which are then analyzed by a time of flight (TOF) mass spectrometer [58]. CyTOF allows close to 100 mass detection channels on a single cell and due to absence of most stable metal isotopes in biological samples, the background signal is minimum with this approach. CyTOF platform might enhance and accelerate cellular and functional biomarker discovery in different diseases including melanoma.

\section{Biomarker Discovery in Melanoma}

High rates of metastasis make it difficult to treat melanoma and result in poor prognosis, especially for patients with advanced disease. Overcoming this challenge requires the design of more targeted therapeutic approaches, which will need to utilize more precise staging and disease characterization methods. Proteomics has emerged as an essential tool to identify new biomarkers that can distinguish melanoma subtypes [20], determine drug resistance status [59] and define stages of melanoma progression [60]. Highly sensitive and specific biomarkers are also key to understanding molecular mechanisms involved in melanoma development and the acquisition of metastatic potential.

\section{Serological Biomarkers}

Serum analysis is cost effective and benefits from an easily accessible sample source that can be obtained from large numbers of patients. Serum analysis seeks to allow the detection of proteins that are secreted by tumors into the bloodstream, which can then serve as biomarkers to help detect the existence of cancer. Serum biomarkers have been shown to possess physiological and pathological relevance to melanoma progression status, and have helped advance our understanding of melanoma onset, progression and response to therapeutics.

Serological biomarkers have been found particularly helpful in determining prognosis in early-stage melanomas. The melanocyte differentiation antigen S100 calcium-binding protein B (S100 beta) and melanoma-inhibitory activity (MIA) are routinely used for early detection of melanoma $[61,62]$. Both proteins correlate well with tumor load. Damude et al., have reported an association of serum S-100 beta levels with residual tumor load, and pre-operative levels of S-100 beta were found to be a strong predictive factor for non-sentinel nodes positivity in patients assigned to undergo complete lymph node dissection [63].

In addition to serological levels, exosomal concentrations of MIA and S100 beta were also found to correlate positively with melanoma progression [64]. MIA and S-100 beta, along with osteopontin (OPN), were also found to correlate with metastatic capabilities of uveal melanomas [65]. Serological levels of the cell adhesion molecule vitronectin and the antimicrobial protein dermcidin were shown to correlate with metastatic progression of early stage melanomas [66]. Recently, a combination of serum biomarker 5 S-cysteinyldopa and circulating melanoma cells (CMC) were used to detect melanoma metastasis [67]. Additionally, an elevated serum level of melanocytespecific secreted glycoprotein (ME20-S) was associated with high tumor burden and advanced disease [68]. Another marker that tracks well with prognosis, tumor burden and disease progression in melanoma patients is the ratio of serum L-dopa to serum L-tyrosine [69]. Ugurel et al., found a positive correlation between serum angiogenic factors vascular endothelial growth factor (VEGF), basic fibroblast growth factor (BFGF) and IL-8 with melanoma progression and survival [70]. Boyano et al., and Nemunaitis et al., documented the correlation of IL-10 with poor survival and advanced disease in melanoma patients $[71,72] . \mathrm{LDH}$ is another strong prognostic biomarker associated with 
advanced melanoma [73]. However, in another study, acute phase C-reactive protein (CRP) has been suggested to be superior to $\mathrm{LDH}$ as a serum marker for detection of stage IV melanoma [74]. In this study, LDH failed to discriminate stage IV melanoma patients from stage I, II or III patients. However, CRP showed much more sensitivity and specificity in diagnosing melanoma patients with stage IV entry. It was suggested that excision of primary melanoma and CRP measurements might allow early detection of metastasis.

More recently, a study by Lugowska et al., evaluated the clinical utility of VEGF, matrix metallopeptidases 2 and 9 (MMP-2 and MMP9), TIMP metallopeptidase inhibitor 1 (TIMP-1) and human cartilage glycoprotein-39 (YKL-40). They found that high TIMP-1 serum levels could predict poor prognosis. YKL-40 was found to be associated with ulceration of primary melanoma tumors. No significant correlations for VEGF, MMP-2 or MMP-9 were detected in either early or late stage melanoma [75].

Biomarkers have demonstrated much utility in determining patient response likelihoods, which is critical to ensure proper design of individual patient treatment plans. For example, ipilimumab has achieved durable response rates in melanoma patients, but not all patients respond to this therapy. A high frequency of myeloid-derived suppressor cells (MDSCs) and high serum IL-6 levels, which are both indicative of immune suppressive tumor environment, correlated with ipilimumab treatment failure in stage IV melanoma patients [76]. By analyzing NY-ESO-1 (a cancer/testis antigen) serum antibody levels in ipilimumab-treated melanoma patients, it was shown that NY-ESO-1 seropositive patients had a greater likelihood of therapeutic benefit in response to treatment. However, some NY-ESO-1 seropositive patients failed to achieve clinical benefit in the absence of CD8 $\mathrm{T}$ cell responses [77].

Baseline serum levels of LDH were also found to be a predictor of durable response to ipilimumab treatment in metastatic melanoma [78]. Similarly, serum LDH levels in patients treated with antiPD-1 therapy correlated negatively with overall survival (OS) [79]. Low neutrophil levels in metastatic melanoma patients treated with ipilimumab correlated with high OS [80]. Elevated chemokine (C-X-C motif) ligand 11 (CXCL11) and soluble MHC class I polypeptiderelated chain A (sMICA) were associated with poor OS in patients with metastatic melanoma post-ipilimumab treatment [81]. Serum levels of soluble oncoprotein c-MET have also been shown to track well with metastasis in uveal melanoma [82]. In general, serum based biomarkers are really helpful in predicting the disease progression and response to a therapeutic intervention.

\section{Cell- or Tissue-based Biomarkers}

Serum analysis is ideal for detecting protein biomarkers expressed at levels sufficient for detection. However, detection of weakly expressed proteins is not possible with current serological techniques. Alternatively, cell culture and tissue specimen analysis can bypass this limitation. Cancer cell lines can be used for initial investigation of prognostic markers, since they are cost effective, gene manipulations are easy with them, and they are helpful in mechanistic investigations. Results obtained from these methods, however, must be validated using patient samples in order to determine physiological significance. The use of human tissues is preferred, due to its greater physiological relevance; however, this can be challenging because of limited availability. Another complication of using human tissue samples is variability amongst samples obtained from different patients.

Qendro et al., have profiled the proteomes of different melanoma cell lines from different stages of melanoma progression. They reported nestin and vimentin as predictive biomarkers for melanoma aggressiveness [20]. Using a genome-wide expression analysis of a lymph node metastasis-derived cell line and comparing it with a cerebrotropic derivative, Jilaveanu et al., identified an association of pleckstrin homology domain-containing family A member 5 (PLEKHA5) with CNS dissemination and homing [83].

Proteomic analyses have also been very useful in identifying predictive markers for therapeutic resistance. In melanoma patients, association of elevated levels of calcium binding protein S100A13 were observed in response to chemotherapy with dacarbazine and temozolomide [84]. Nawarak et al., performed proteomic analysis of A375 human malignant melanoma cells in response to arbutin treatment. They observed upregulation of 14-3-3G, VDAC-1 and p53. Reciprocally, ENPL, ENOA, IMDH2, PRDX1 and VIME were downregulated. Altogether, these proteins were found to be involved in suppression of carcinogenesis [32].

Liquid chromatography-multiple reaction monitoring mass spectrometry (LC-MRM) was utilized to evaluate anti-tumor activity of the HSP90 inhibitor XL888 against BRAF mutant melanoma both in vivo and in vitro [85]. Mactier et al., identified signatures corresponding to survival outcomes of advanced stage III melanoma patients. This study identified 21 potential survival biomarkers involved in protein metabolism, nucleic acid metabolism, angiogenesis, etc. Among the various biomarkers identified, periostin, HSP90 ßeta, poly ADP-ribose polymerase were most promising [49].

Byrum et al., conducted a large proteome study and identified 5 proteins [heterogeneous nuclear ribonucleoprotein L (HNRPL), ferritin light chain (FTL), cytochrome c oxidase subunit 4 isoform 1 (COX4I1), decorin $(\mathrm{DCN})$, lumican (LUM)] that were differentially expressed between primary and metastatic melanomas [86]. MINERVA/FAM129B was identified as a target of phosphorylation by B-raf signaling using a phosphoproteome approach in melanoma [87]. This is really helpful in understanding the molecular mechanism(s) involved in B-raf mutation in melanoma patients. Phosphoproteome analysis was also used to profile the kinase landscape for resistance to B-raf inhibitor therapy in melanoma. It was shown that after transitioning to a drug resistant phenotype, abundance of phosphopeptides associated with cytoskeletal regulation, protein kinase C, IGF signaling, GTP/GDP exchange and melanoma maturation were divergent [88]. In another study using a phosphoproteome approach, ROCK1, a serine/threonine kinase, was identified as a potential drug target for BRAF mutant melanoma [89].

By applying proteomic analysis to melanoma exosomes, it was shown that exosome protein composition correlates strongly with tumor aggressiveness. Exosomes from aggressive cells were found to contain proteins involved in motility, angiogenesis and immune responsiveness, whereas proteins from less aggressive cells were less abundant [90]. A study by Crabb et al., determined collagen alpha$3(\mathrm{VI})$ and heat shock protein beta- 1 as potential predictive biomarkers for uveal melanoma metastasis [37].

Utilizing tissues from melanoma patients at different stages of tumor progression, it was shown that melanogenesis associated transcription factor (MITF) was expressed in all stages of tumor progression. Bcl-2 expression levels were found to be reduced with tumor progression. In contrast to melanoma antigen recognized by T cells 1 (MART-1), which showed no differential expression, human melanoma black 45 (HMB45), a antibody that reacts against antigen present in melanocytic tumors, expresses higher in primary and metastatic melanomas as 
Citation: Mittal P, Jain M (2016) Proteomics: An Indispensable Tool for Novel Biomarker Identification in Melanoma. J Data Mining Genomics Proteomics 7: 204. doi:10.4172/2153-0602.1000204

Page 7 of 10

\begin{tabular}{|c|c|c|c|c|}
\hline Author & Year & Sample & Proteomic platform & Finding regarding Biomarker associated with melanoma \\
\hline Caputo et al. [38] & 2011 & $\begin{array}{l}\text { Human melanoma cell } \\
\text { lines and melanocytes }\end{array}$ & 2D PAGE and LC-MS/MS & $\begin{array}{l}\text { Galectin-1, inosine-5'-monophosphate dehydrogenase } 2 \text {, serine/threonine- } \\
\text { protein phosphatase } 2 A 65 \mathrm{kDa} \text { regulatory subunit } A \text { alpha isoform, protein } \\
\text { DJ-1, cyclophilin A and cofilin-1 were upregulated in melanoma cell lines } \\
\text { as compared to melanocytes }\end{array}$ \\
\hline Liu et al. [55] & 2011 & $\begin{array}{l}\text { Melanoma cells with high } \\
\text { or low metastatic abilities }\end{array}$ & $\begin{array}{l}\text { SILAC, nanospray tandem mass } \\
\text { spectrometry }\end{array}$ & $\begin{array}{l}\text { CDCP1 is differentially expressed transmembrane protein in tumors with } \\
\text { high metastasis }\end{array}$ \\
\hline Martin et al. [40] & 2011 & Plasma & SPE-LC-ESI-MS/MS & $\begin{array}{l}\text { Plasma levels of melanoma biomarker MS5-S-cysteinyldopa was } \\
\text { determined }\end{array}$ \\
\hline Yuan et al. [78] & 2011 & Serum & ELISA & $\begin{array}{l}\text { NY-ESO-1 seropositive patients with associated CD8 }{ }^{+} T \text { cells experiences } \\
\text { significant survival advantage along with frequent clinical benefit than } \\
\text { those with undetectable } C D 8^{+} T \text { cells }\end{array}$ \\
\hline Jones et al. [13] & 2012 & Serum & $\begin{array}{l}\text { Serum/urine electrophoresis and } \\
\text { serum-free light chain detection, } \\
\text { blood count and biochemical } \\
\text { profile, microarray }\end{array}$ & $\begin{array}{l}\text { microRNAs miR-720, miR-1308 and miR-1246 were found to have } \\
\text { potential as diagnostic biomarkers in myeloma }\end{array}$ \\
\hline Linge et al. [35] & 2012 & Melanoma tissue & 2-D DIGE, MS, IHC & $\begin{array}{l}\text { Increased expression of PDIA3, VIM/HEXA, SELENBP1, ENO1, CAPZA1, } \\
\text { ERP29, TPI1, PARK7, and FABP3 along with decreased expression } \\
\text { EIF2S, PSMA3, RPSA, TUBB and TUBA1B in metastasized uveal } \\
\text { melanomas compared to non-metastatic one }\end{array}$ \\
\hline Byrum et al. [87] & 2013 & Melanoma Tissue & LC-MS/MS & $\begin{array}{l}171 \text { proteins were found to be varying in their expression levels among } \\
\text { benign nevi, primary melanoma, and metastatic melanoma suggesting } \\
\text { that molecular pathways involved with tumor cell proliferation, motility and } \\
\text { apoptosis are mis-regulated in melanoma }\end{array}$ \\
\hline Delyon et al. [14] & 2013 & Serum & Blood count & $\begin{array}{l}\text { Higher lymphocyte and eosinophil counts at the time of the second } \\
\text { ipilimumab infusion appears well correlated with an improved OS }\end{array}$ \\
\hline Azimi et al. [85] & 2014 & $\begin{array}{l}\text { Frozen lymph node } \\
\text { metastasis }\end{array}$ & $\begin{array}{l}\text { iTRAQ labeling, narrow-range IEF, } \\
\text { MS, Immunoblotting, IHC }\end{array}$ & $\begin{array}{l}\text { S100A13 was highly upregulated in non-responders to dacarbazine (DTIC) } \\
\text { or temozolomide (TMZ) chemotherapy in case of cutaneous malignant } \\
\text { melanoma }\end{array}$ \\
\hline Mactier S et al. [50] & 2014 & Tissue & iTRAQ, 2DLC-MS/MS, 2-D DIGE & $\begin{array}{l}\text { Poor prognosis in patients with increased expression of proteins involved in } \\
\text { protein metabolism, nucleic acid metabolism, angiogenesis, deregulation } \\
\text { of cellular energetics and methylation processes and decreased levels of } \\
\text { proteins involved in apoptosis and immune response }\end{array}$ \\
\hline Qendro et al. [20] & 2014 & Tissue, cell lines & LC-MS/MS & $\begin{array}{l}\text { Higher levels of expression of nestin and vimentin correlated with } \\
\text { aggressiveness of melanoma }\end{array}$ \\
\hline Rebecca et al. [86] & 2014 & Melanoma cell lines & GeLC-MRM, flow cytometry & $\begin{array}{l}\text { HSP90 inhibition was found to be associated with decreased expression of } \\
\text { multiple receptor tyrosine kinases, modules in the PI3K/AKT/mammalian target } \\
\text { of rapamycin pathway and MAPK/CDK4 signaling axis. vemurafenib resistance } \\
\text { resulted in inhibition of PI3K/AKT signaling in acquired BRAF mutant melanoma }\end{array}$ \\
\hline Barisione et al. [83] & 2015 & Serum, cell lines & ELISA & $\begin{array}{l}\text { Serum levels of sc-Met can sever as a novel biomarker candidate in } \\
\text { metastatic uveal melanoma as it was found to be significantly elevated in } \\
\text { uveal melanoma patients with metastasis compared to patients with non- } \\
\text { metastatic cancer }\end{array}$ \\
\hline Bande et al. [69] & 2015 & Serum & ELISA, biochemical assays & $\begin{array}{l}\text { Elevated ME20-S serum levels were associated with tumor burden and } \\
\text { advanced stages of uveal melanoma }\end{array}$ \\
\hline Crabb et al. [37] & 2015 & $\begin{array}{l}\text { Metastatic and non- } \\
\text { metastatic uveal primary } \\
\text { melanoma tumors }\end{array}$ & $\begin{array}{l}\text { iTRAQ, cation exchange } \\
\text { chromatography, LC- MS/MS }\end{array}$ & $\begin{array}{l}\text { Collagen alpha- } 3(\mathrm{VI}) \text { and heat shock protein beta-1 were identified as } \\
\text { candidate biomarkers of uveal melanoma metastasis }\end{array}$ \\
\hline Guan et al. [33] & 2015 & $\begin{array}{l}\text { Primary melanoma } \\
\text { tissue, metastatic lymph } \\
\text { node tissue, serum, } \\
\text { melanoma cell lines }\end{array}$ & 2DE, MS, IHC & $\begin{array}{l}\text { MDA-9 and GRP78 may serve as potential diagnostic biomarkers for early } \\
\text { detection of melanoma metastasis }\end{array}$ \\
\hline Koguchi et al. [82] & 2015 & Serum & Multiplexed immunoassay, ELISA & $\begin{array}{l}\text { Pretreatment levels of CXCL11 and SMICA may represent predictors of } \\
\text { survival benefit after ipilimumab treatment but not vaccine treatment as } \\
\text { higher baseline levels of these were found to be associated with poor OS } \\
\text { in melanoma patients with metastasis }\end{array}$ \\
\hline Lazar et al. [91] & 2015 & Metastatic cell lines & Nano-LC-MS/MS & $\begin{array}{l}\text { Protein composition of melanoma exosomes depends on the cell's } \\
\text { aggressivity and exosomal contents influence the behavior of tumor cells } \\
\text { and their microenvironment }\end{array}$ \\
\hline Lugowska et al. [76] & 2015 & Serum & ELISA & $\begin{array}{l}\text { In melanoma patients at stages I-III, the high serum concentrations of } \\
\text { TIMP-1 predicts adverse prognosis and YKL- } 40 \text { was associated with } \\
\text { ulceration of primary tumor }\end{array}$ \\
\hline Parker et al. [89] & 2015 & Melanoma cell lines & Isobaric labeling, LC-MS/MS & $\begin{array}{l}\text { The abundance of phosphopeptide sites associated with cytoskeletal } \\
\text { regulation, GTP/GDP exchange, protein kinase C, IGF signaling, and } \\
\text { melanosome maturation were highly divergent in response to resistance } \\
\text { to BRAF inhibitor therapy }\end{array}$ \\
\hline Valpione et al. [81] & 2015 & Serum & Cytoflurometry & $\begin{array}{l}\text { Higher baseline LDH and neutrophils levels were associated with worse } \\
\text { prognosis in melanoma patients treated with ipilimumab }\end{array}$ \\
\hline Welinder et al. [57] & 2015 & Lymph node metastases & $\begin{array}{l}\text { Cation exchange chromatography, } \\
\text { LC-MS/MS, Deep protein } \\
\text { sequencing }\end{array}$ & $\begin{array}{l}\text { Build a metastatic melanoma protein sequence database by identifying } \\
\text { more than } 5000 \text { unique proteins involved in melanoma metastasis }\end{array}$ \\
\hline
\end{tabular}




\begin{tabular}{|l|l|l|l|l|}
\hline Alegre et al. [65] & 2016 & Serum & ELISA & $\begin{array}{l}\text { Exosomal concentrations of MIA and S100B serve as prognostic and } \\
\text { diagnostic markers for melanoma }\end{array}$ \\
\hline Bjoern et al. [77] & 2016 & Serum & $\begin{array}{l}\text { Malignant melanoma patients with increased absolute lymphocyte count } \\
\text { and T cell activation responded to ipilimumab treatment better. Higher } \\
\text { frequency of MDSCs along with high levels of IL6 reduced chance of } \\
\text { response to therapy }\end{array}$ \\
\hline Damude et al. [64] & 2016 & $\begin{array}{l}\text { Sentinal lymph node } \\
\text { biopsy }\end{array}$ & IHC & $\begin{array}{l}\text { The preoperatively levels of S-100B is the strongest predictor for non- } \\
\text { sentinel nodes positivity in patients planning for subsequent lymph node } \\
\text { dissection }\end{array}$ \\
\hline Hida et al. [68] & 2016 & Serum & Fluorophore conjugated antibodies & $\begin{array}{l}\text { Circulating melanoma cells can complement the efficacy of 5-S-CD to } \\
\text { detect metastasis and hence they can serve as a prognostic marker }\end{array}$ \\
\hline Kwek et al. [5] & 2016 & Serum & ELISA & $\begin{array}{l}\text { Lower levels of CD4 }{ }^{+} \text {effector cells, high levels of PD-1 expressing CD8 } \\
\text { cells correlated with melanoma control }\end{array}$ \\
\hline $\begin{array}{l}\text { Ortega-martinez et } \\
\text { al. [67] }\end{array}$ & 2016 & Serum & ELISA, MS & $\begin{array}{l}\text { Expression levels of vitronectin and dermcidin were found to be associated } \\
\text { with the metastatic progression in patients with early-stage melanoma }\end{array}$ \\
\hline Weide et al. [12] & 2016 & Blood & $\begin{array}{l}\text { Full blood count and biochemical } \\
\text { profiling }\end{array}$ & $\begin{array}{l}\text { High relative eosinophil and lymphocyte count, low LDH and absence of } \\
\text { metastasis other than soft-tissue/lung related to high OS }\end{array}$ \\
\hline
\end{tabular}

Table 1: Melanoma biomarkers predicted using proteomics approach.

compared to nevi. C-Kit, on the other hand, was found to increase upon tumor progression from nevus to primary lesion, but decrease upon the acquisition of metastatic potential [91].

Melanoma tissue lysates were used to identify miR10b as a novel prognostic microRNA for melanoma progression. Expression profiling of miR10b showed a trend towards increased expression between primary melanomas and their corresponding metastatic tumors [92]. Sengupta et al. have reported some of the findings regarding melanoma biomarkers with emphasis on different proteomic techniques used and findings from those studies [93]. However, we included a table (Table 1) that compiles up recent proteomic studies that have identified various melanoma specific biomarkers.

\section{Future Perspective and Concluding Remarks}

Proteome analysis has emerged as a powerful tool for analyzing highly complex samples from melanoma patients. It has played an important role in the identification of numerous biomarkers associated with early disease prognosis, progression, metastasis, therapeutic efficacy, resistance mechanisms, and treatment-related toxicities. There is a pressing need for definitive biomarkers to allow for proper staging of melanoma lesions. The use of proteomics has gained momentum due to its sensitivity, accuracy, speed, and throughput, as well as the development of powerful analytical tools and software available. To date, however, $\mathrm{LDH}$ is the only biomarker used clinically. Reproducibility of proteomic studies remains a concern, and thus validation of biomarker identification necessitates investigation of large patient cohorts. Additionally, many candidate biomarkers discovered might turn out not to be directly related to tumor biology. A limitation in serum detection thresholds also remains a hurdle; however, more sensitive and sophisticated protein quantification techniques capable of detecting femtomolar concentrations of protein are being developed. Advances in proteomic studies on the horizon will contribute to a better understanding of drug mechanism of action in response to various therapies [94]. This understanding could eventually help guide the design of the next generation cancer therapeutics, as well as play an important role in implementing personalized medicine approaches. The discovery and utilization of biomarkers will inevitably help shape the future of melanoma diagnosis and treatment, and proteomic analysis will play an essential part in achieving this goal.

\section{Acknowledgements}

We would like to acknowledge Mr. Joseph Ryan for helpful discussions and proofreading the manuscript.

\section{References}

1. Kulichova D, Danova J, Kunte C, Ruzicka T, Celko AM (2014) Risk factors for malignant melanoma and preventive methods. Cutis 94: 241-248.

2. Neuschmelting V, Lockau H, Ntziachristos V, Grimm J, Kircher MF (2016) Lymph Node Micrometastases and In-Transit Metastases from Melanoma: In Vivo Detection with Multispectral Optoacoustic Imaging in a Mouse Model. Radiology.

3. Ennen M, Keime C, Kobi D, Mengus G, Lipsker D, et al. (2015) Single-cel gene expression signatures reveal melanoma cell heterogeneity. Oncogene 34: $3251-3263$.

4. Mittal P, St Rose MC, Wang X, Ryan JM, Wasser JS, et al. (2015) TumorUnrelated CD4 T Cell Help Augments CD134 plus CD137 Dual Costimulation Tumor Therapy. J Immunol 195: 5816-5826.

5. Kwek SS, Kahn J, Greaney SK, Lewis J, Cha E, et al. (2016) GM-CSF and ipilimumab therapy in metastatic melanoma: Clinical outcomes and immunologic responses. Oncoimmunology 5: e1101204.

6. Larkin J, Chiarion-Sileni V, Gonzalez R, Grob JJ, Cowey CL, et al. (2015) Combined Nivolumab and Ipilimumab or Monotherapy in Untreated Melanoma. N Engl J Med 373: 23-34.

7. Mayeux R (2004) Biomarkers: potential uses and limitations. NeuroRx 1: 182-188.

8. Walther A, Johnstone E, Swanton C, Midgley R, Tomlinson I, et al. (2009) Genetic prognostic and predictive markers in colorectal cancer. Nat Rev Cancer 9: 489-499.

9. Davies H, Bignell GR, Cox C, Stephens P, Edkins S, et al. (2002) Mutations of the BRAF gene in human cancer. Nature 417: 949-954

10. Gong M, Dong W, Shi Z, Xu Y, Ni W, et al. (2012) Genetic polymorphisms of GSTM1, GSTT1, and GSTP1 with prostate cancer risk: a meta-analysis of 57 studies. PLoS One 7: e50587.

11. Samzadeh M, Hasanzad $M$, Jamaldini $S H$, Haghdoost $A A$, Afshari $M$, et al (2012) Association of G/A polymorphism, rs266882, in AREI region of the prostate-specific antigen gene with prostate cancer risk and clinicopathological features. Urol J 9: 691-699.

12. Weide B, Martens A, Hassel JC, Berking C, Postow MA, et al. (2016) Baseline biomarkers for outcome of melanoma patients treated with pembrolizumab. Clin Cancer Res.

13. Jones Cl, Zabolotskaya MV, King AJ, Stewart HJ, Horne GA, et al. (2012) Identification of circulating microRNAs as diagnostic biomarkers for use in multiple myeloma. Br J Cancer 107: 1987-1996.

14. Delyon J, Mateus C, Lefeuvre D, Lanoy E, Zitvogel L, et al. (2013) Experience in daily practice with ipilimumab for the treatment of patients with metastatic melanoma: an early increase in lymphocyte and eosinophil counts is associated with improved survival. Ann Oncol 24: 1697-1703.

15. Haqq C, Nosrati M, Sudilovsky D, Crothers J, Khodabakhsh D, et al. (2005) The gene expression signatures of melanoma progression. Proc Natl Acad Sc U S A 102: 6092-6097. 
Citation: Mittal P, Jain M (2016) Proteomics: An Indispensable Tool for Novel Biomarker Identification in Melanoma. J Data Mining Genomics Proteomics 7: 204. doi:10.4172/2153-0602.1000204

16. Trolet J, Hupe P, Huon I, Lebigot I, Decraene C, et al. (2009) Genomic profiling and identification of high-risk uveal melanoma by array $\mathrm{CGH}$ analysis of primary tumors and liver metastases. Invest Ophthalmol Vis Sci 50: 2572-2580.

17. Marzese DM, Huynh JL, Kawas NP, Hoon DS (2014) Multi-platform Genome-wide Analysis of Melanoma Progression to Brain Metastasis. Genom Data 2: 150-152.

18. Srinivas PR, Srivastava S, Hanash S, Wright GL Jr (2001) Proteomics in early detection of cancer. Clin Chem 47: 1901-1911.

19. Celis JE, Ostergaard M, Jensen NA, Gromova I, Rasmussen HH, et al. (1998) Human and mouse proteomic databases: novel resources in the protein universe. FEBS Lett 430: 64-72.

20. Qendro V, Lundgren DH, Rezaul K, Mahony F, Ferrell N, et al. (2014) Largescale proteomic characterization of melanoma expressed proteins reveals nestin and vimentin as biomarkers that can potentially distinguish melanoma subtypes. J Proteome Res 13: 5031-5040.

21. Chen El, Yates JR 3rd (2007) Cancer proteomics by quantitative shotgun proteomics. Mol Oncol 1: 144-159.

22. Soldi M, Cuomo A, Bonaldi T (2014) Improved bottom-up strategy to efficiently separate hypermodified histone peptides through ultra-HPLC separation on a bench top Orbitrap instrument. Proteomics 14: 2212-2225.

23. Vuckovic D, Dagley LF, Purcell AW, Emili A (2013) Membrane proteomics by high performance liquid chromatography-tandem mass spectrometry: Analytical approaches and challenges. Proteomics 13: 404-423.

24. Matharoo-Ball B, Ratcliffe L, Lancashire L, Ugurel S, Miles AK, et al. (2007) Diagnostic biomarkers differentiating metastatic melanoma patients from healthy controls identified by an integrated MALDI-TOF mass spectrometry/ bioinformatic approach. Proteomics Clin Appl 1: 605-620.

25. Martin GB, Chiap P, Paquet P, Pierard G, de Tullio P, et al. (2007) Development of a mass spectrometry method for the determination of a melanoma biomarker, 5 -S-cysteinyldopa, in human plasma using solid phase extraction for sample clean-up. J Chromatogr A 1156: 141-148.

26. Fenn JB, Mann M, Meng CK, Wong SF, Whitehouse CM (1989) Electrospray ionization for mass spectrometry of large biomolecules. Science 246: 64-71.

27. Hillenkamp F, Karas M, Beavis RC, Chait BT (1991) Matrix-assisted laser desorption/ionization mass spectrometry of biopolymers. Anal Chem 63: 1193A-1203A.

28. Wright PC, Noirel J, Ow SY, Fazeli A (2012) A review of current proteomics technologies with a survey on their widespread use in reproductive biology investigations. Theriogenology 77: 738 e752-765 e752.

29. Yates JR 3rd (2000) Mass spectrometry. From genomics to proteomics. Trends Genet 16: 5-8.

30. Greco M, Mitri MD, Chiriaco F, Leo G, Brienza E, et al. (2009) Serum proteomic profile of cutaneous malignant melanoma and relation to cancer progression: association to tumor derived alpha- $\mathrm{N}$-acetylgalactosaminidase activity. Cancer Lett 283: 222-229.

31. Chen J, Wei D, Zhuang H, Qiao Y, Tang B, et al. (2011) Proteomic screening of anaerobically regulated promoters from Salmonella and its antitumor applications. Mol Cell Proteomics 10: M111.009399.

32. Nawarak J, Huang-Liu R, Kao SH, Liao HH, Sinchaikul S, et al. (2009) Proteomics analysis of $A 375$ human malignant melanoma cells in response to arbutin treatment. Biochim Biophys Acta 1794: 159-167.

33. Guan M, Chen X, Ma Y, Tang L, Guan L, et al. (2015) MDA-9 and GRP78 as potential diagnostic biomarkers for early detection of melanoma metastasis. Tumour Biol 36: 2973-2982.

34. Alban A, David SO, Bjorkesten L, Andersson C, Sloge E, et al. (2003) A novel experimental design for comparative two-dimensional gel analysis: twodimensional difference gel electrophoresis incorporating a pooled internal standard. Proteomics 3: 36-44.

35. Linge A, Kennedy S, O'Flynn D, Beatty S, Moriarty P, et al. (2012) Differential expression of fourteen proteins between uveal melanoma from patients who subsequently developed distant metastases versus those who did Not. Invest Ophthalmol Vis Sci 53: 4634-4643.

36. Li M, Zhang B, Sun B, Wang X, Ban X, et al. (2010) A novel function for vimentin: the potential biomarker for predicting melanoma hematogenous metastasis. $J$ Exp Clin Cancer Res 29: 109
37. Crabb JW, Hu B, Crabb JS, Triozzi P, Saunthararajah Y, et al. (2015) iTRAQ Quantitative Proteomic Comparison of Metastatic and Non-Metastatic Uvea Melanoma Tumors. PLoS One 10: e0135543.

38. Caputo E, Maiorana L, Vasta V, Pezzino FM, Sunkara S, et al. (2011) Characterization of human melanoma cell lines and melanocytes by proteome analysis. Cell Cycle 10: 2924-2936.

39. Huang SK, Darfler MM, Nicholl MB, You J, Bemis KG, et al. (2009) LC/MS-based quantitative proteomic analysis of paraffin-embedded archival melanomas reveals potential proteomic biomarkers associated with metastasis. PLoS One 4: e4430.

40. Martin G, Mansion F, Houbart V, Paquet P, Rorive A, et al. (2011) Pre-study and in-study validation of a SPE-LC-MS-MS method for the determination of 5-S-cysteinyldopa, a melanoma biomarker, in human plasma. Talanta 84: 280-286.

41. Yao X, Freas A, Ramirez J, Demirev PA, Fenselau C (2001) Proteolytic 180 labeling for comparative proteomics: model studies with two serotypes of adenovirus. Anal Chem 73: 2836-2842.

42. Stockwin LH, Blonder J, Bumke MA, Lucas DA, Chan KC et al. (2006) Proteomic analysis of plasma membrane from hypoxia-adapted malignant melanoma. J Proteome Res 5: 2996-3007.

43. Thelen JJ, Peck SC (2007) Quantitative proteomics in plants: choices in abundance. Plant Cell 19: 3339-3346.

44. Jay J Thelen, Scott C Peck (2007) Quantitative Proteomics in Plants: Choices in Abundance. The Plant Cell 19: 3339-3346.

45. Kang UB, Ahn Y, Lee JW, Kim YH, Kim J, et al. (2010) Differential profiling of breast cancer plasma proteome by isotope-coded affinity tagging method reveals biotinidase as a breast cancer biomarker. BMC Cancer 10: 114 .

46. Leroy B, Rosier C, Erculisse V, Leys N, Mergeay M, et al. (2010) Differential proteomic analysis using isotope-coded protein-labeling strategies: comparison, improvements and application to simulated microgravity effect on Cupriavidus metallidurans $\mathrm{CH} 34$. Proteomics 10: 2281-2291.

47. Fleron M, Greffe Y, Musmeci D, Massart AC, Hennequiere V, et al. (2010) Novel post-digest isotope coded protein labeling method for phospho- and glycoproteome analysis. J Proteomics 73: 1986-2005.

48. Rainczuk A, Condina M, Pelzing M, Dolman S, Rao J, et al. (2013) The utility of isotope-coded protein labeling for prioritization of proteins found in ovarian cancer patient urine. J Proteome Res 12: 4074-4088.

49. Ross PL, Huang YN, Marchese JN, Williamson B, Parker K, et al. (2004 Multiplexed protein quantitation in Saccharomyces cerevisiae using aminereactive isobaric tagging reagents. Mol Cell Proteomics 3: 1154-1169.

50. Mactier S, Kaufman KL, Wang P, Crossett B, Pupo GM, et al. (2014) Protein signatures correspond to survival outcomes of AJCC stage III melanoma patients. Pigment Cell Melanoma Res 27: 1106-1116.

51. Thompson A, Schafer J, Kuhn K, Kienle S, Schwarz J, et al. (2003) Tandem mass tags: a novel quantification strategy for comparative analysis of complex protein mixtures by MS/MS. Anal Chem 75: 1895-1904.

52. Xiao H, Zhang Y, Kim Y, Kim S, Kim JJ, et al. (2016) Differential Proteomic Analysis of Human Saliva using Tandem Mass Tags Quantification for Gastric Cancer Detection. Sci Rep 6: 22165.

53. Ramasamy P, Murphy CC, Clynes M, Horgan N, Moriarty P, et al. (2014) Proteomics in uveal melanoma. Exp Eye Res 118: 1-12.

54. Ong SE, Blagoev B, Kratchmarova I, Kristensen DB, Steen H, et al. (2002) Stable isotope labeling by amino acids in cell culture, SILAC, as a simple and accurate approach to expression proteomics. Mol Cell Proteomics 1: 376-386.

55. Liu H, Ong SE, Badu-Nkansah K, Schindler J, White FM, et al. (2011) CUB domain-containing protein 1 (CDCP1) activates Src to promote melanoma metastasis. Proc Natl Acad Sci U S A 108: 1379-1384.

56. Ippel JH, Pouvreau L, Kroef T, Gruppen H, Versteeg G, et al. (2004) In vivo uniform (15) $\mathrm{N}$-isotope labelling of plants: using the greenhouse for structural proteomics. Proteomics 4: 226-234.

57. Welinder C, Pawlowski K, Sugihara Y, Yakovleva M, Jonsson G, et al. (2015) A protein deep sequencing evaluation of metastatic melanoma tissues. PLoS One 10: e0123661.

58. Chung L, Moore K, Phillips L, Boyle FM, Marsh DJ, et al. (2014) Novel serum protein biomarker panel revealed by mass spectrometry and its prognostic value in breast cancer. Breast Cancer Res 16: R63. 
Citation: Mittal P, Jain M (2016) Proteomics: An Indispensable Tool for Novel Biomarker Identification in Melanoma. J Data Mining Genomics Proteomics 7: 204. doi:10.4172/2153-0602.1000204

59. Bendall SC, Simonds EF, Qiu P, Amir el AD, Krutzik PO, et al. (2011) Singlecell mass cytometry of differential immune and drug responses across a human hematopoietic continuum. Science 332: 687-696.

60. Paulitschke V, Eichhoff O, Cheng PF, Levesque MP, Holler C (2016) Proteomics approaches to understanding mitogen-activated protein kinase inhibitor resistance in melanoma. Curr Opin Oncol 28: 172-179.

61. Bernard K, Litman E, Fitzpatrick JL, Shellman YG, Argast G, et al. (2003) Functional proteomic analysis of melanoma progression. Cancer Res 63 6716-6725.

62. Krahn G, Kaskel P, Sander S, Waizenhofer PJ, Wortmann S, et al. (2001) S100 beta is a more reliable tumor marker in peripheral blood for patients with newly occurred melanoma metastases compared with MIA, albumin and lactatedehydrogenase. Anticancer Res 21: 1311-1316.

63. Garbe C, Leiter U, Ellwanger U, Blaheta HJ, Meier F, et al. (2003) Diagnostic value and prognostic significance of protein S-100beta, melanoma-inhibitory activity, and tyrosinase/MART-1 reverse transcription-polymerase chain reaction in the follow-up of high-risk melanoma patients. Cancer 97: 1737-1745.

64. Damude S, Hoekstra HJ, Bastiaannet E, Muller Kobold AC, Kruijff S, et al. (2016) The predictive power of serum S-100B for non-sentinel node positivity in melanoma patients. Eur J Surg Oncol 42: 545-551.

65. Alegre E, Zubiri L, Perez-Gracia JL, Gonzalez-Cao M, Soria L, et al. (2016) Circulating melanoma exosomes as diagnostic and prognosis biomarkers. Clin Chim Acta 454: 28-32.

66. Barak V, Frenkel S, Kalickman I, Maniotis AJ, Folberg R, et al. (2007) Serum markers to detect metastatic uveal melanoma. Anticancer Res 27: 1897-1900.

67. Ortega-Martinez I, Gardeazabal J, Erramuzpe A, Sanchez-Diez A, Cortes J, et al. (2016) Vitronectin and dermcidin serum levels predict the metastatic progression of AJCC I-II early stage melanoma. Int J Cancer.

68. Hida T, Yoneta A, Wakamatsu K, Yanagisawa K, Ishii-Osai Y, et al. (2016) Circulating melanoma cells as a potential biomarker to detect metastasis and evaluate prognosis. Australas J Dermatol 57: 145-149.

69. Bande MF, Santiago M, Mera P, Piulats JM, Blanco MJ, et al. (2015) ME20-S as a Potential Biomarker for the Evaluation of Uveal Melanoma. Invest Ophthalmol Vis Sci 56: 7007-7011.

70. Stoitchkov K, Letellier S, Garnier JP, Bousquet B, Tsankov N, et al. (2003) Evaluation of the serum L-dopa/L-tyrosine ratio as a melanoma marker. Melanoma Res 13: 587-593.

71. Ugurel S, Rappl G, Tilgen W, Reinhold U (2001) Increased serum concentration of angiogenic factors in malignant melanoma patients correlates with tumor progression and survival. J Clin Oncol 19: 577-583.

72. Boyano MD, Garcia-Vazquez MD, Lopez-Michelena T, Gardeazabal J, Bilbao J, et al. (2000) Soluble interleukin-2 receptor, intercellular adhesion molecule-1 and interleukin-10 serum levels in patients with melanoma. $\mathrm{Br} \mathrm{J}$ Cancer 83: 847-852

73. Nemunaitis J, Fong T, Shabe P, Martineau D, Ando D (2001) Comparison of serum interleukin-10 (IL-10) levels between normal volunteers and patients with advanced melanoma. Cancer Invest 19: 239-247.

74. Agarwala SS, Keilholz U, Gilles E, Bedikian AY, Wu J, et al. (2009) LDH correlation with survival in advanced melanoma from two large, randomised trials (Oblimersen GM301 and EORTC 18951). Eur J Cancer 45: 1807-1814.

75. Deichmann M, Kahle B, Moser K, Wacker J, Wust K (2004) Diagnosing melanoma patients entering American Joint Committee on Cancer stage IV, C-reactive protein in serum is superior to lactate dehydrogenase. $\mathrm{Br} \mathrm{J}$ Cancer 91: 699-702.

76. Lugowska I, Kowalska M, Fuksiewicz M, Kotowicz B, Mierzejewska E, et al. (2015) Serum markers in early-stage and locally advanced melanoma. Tumour Biol 36: 8277-8285.

77. Bjoern J, Juul Nitschke N, Zeeberg Iversen T, Schmidt H, Fode K, et al. (2016) Immunological correlates of treatment and response in stage IV malignant melanoma patients treated with Ipilimumab. Oncoimmunology 5: e1100788.

78. Yuan J, Adamow M, Ginsberg BA, Rasalan TS, Ritter E, et al. (2011) Integrated NY-ESO-1 antibody and CD8+ T-cell responses correlate with clinical benefit in advanced melanoma patients treated with ipilimumab. Proc Natl Acad Sci U S A 108: 16723-16728.
79. Kelderman S, Heemskerk B, van Tinteren $\mathrm{H}$, van den Brom RR, Hospers GA et al. (2014) Lactate dehydrogenase as a selection criterion for ipilimumab treatment in metastatic melanoma. Cancer Immunol Immunother 63: 449-458.

80. Diem S, Kasenda B, Spain L, Martin-Liberal J, Marconcini R, et al. (2016) Serum lactate dehydrogenase as an early marker for outcome in patients treated with anti-PD-1 therapy in metastatic melanoma. $\mathrm{Br} \mathrm{J}$ Cancer 114: 256-261.

81. Valpione S, Martinoli C, Fava P, Mocellin S, Campana LG, et al. (2015) Personalised medicine: Development and external validation of a prognostic model for metastatic melanoma patients treated with ipilimumab. Eur J Cancer 51: 2086-2094.

82. Koguchi Y, Hoen HM, Bambina SA, Rynning MD, Fuerstenberg RK, et al. (2015) Serum Immunoregulatory Proteins as Predictors of Overall Survival of Metastatic Melanoma Patients Treated with Ipilimumab. Cancer Res 75: 5084-5092.

83. Barisione G, Fabbi M, Gino A, Queirolo P, Orgiano L, et al. (2015) Potentia Role of Soluble c-Met as a New Candidate Biomarker of Metastatic Uveal Melanoma. JAMA Ophthalmol 133: 1013-1021.

84. Jilaveanu LB, Parisi F, Barr ML, Zito CR, Cruz-Munoz W, et al. (2015) PLEKHA5 as a Biomarker and Potential Mediator of Melanoma Brain Metastasis. Clin Cancer Res 21: 2138-2147.

85. Azimi A, Pernemalm M, Frostvik Stolt M, Hansson J, Lehtio J, et al. (2014) Proteomics analysis of melanoma metastases: association between S100A13 expression and chemotherapy resistance. $\mathrm{Br} \mathrm{J}$ Cancer 110: 2489-2495.

86. Rebecca VW, Wood E, Fedorenko IV, Paraiso KH, Haarberg HE, et al. (2014) Evaluating melanoma drug response and therapeutic escape with quantitative proteomics. Mol Cell Proteomics 13: 1844-1854.

87. Byrum SD, Larson SK, Avaritt NL, Moreland LE, Mackintosh SG, et al. (2013) Quantitative Proteomics Identifies Activation of Hallmark Pathways of Cance in Patient Melanoma. J Proteomics Bioinform 6: 43-50.

88. Old WM, Shabb JB, Houel S, Wang H, Couts KL, et al. (2009) Functional proteomics identifies targets of phosphorylation by B-Raf signaling in melanoma. Mol Cell 34: 115-131.

89. Parker R, Vella LJ, Xavier D, Amirkhani A Parker J, et al. (2015) Phosphoproteomic Analysis of Cell-Based Resistance to BRAF Inhibitor Therapy in Melanoma. Front Oncol 5: 95.

90. Smit MA, Maddalo G, Greig K, Raaijmakers LM, Possik PA, et al. (2014) ROCK1 is a potential combinatorial drug target for BRAF mutant melanoma. Mol Syst Biol 10: 772.

91. Lazar I, Clement E, Ducoux-Petit M, Denat L, Soldan V, et al. (2015) Proteome characterization of melanoma exosomes reveals a specific signature for metastatic cell lines. Pigment Cell Melanoma Res 28: 464-475.

92. Nazarian RM, Prieto VG, Elder DE, Duncan LM (2010) Melanoma biomarker expression in melanocytic tumor progression: a tissue microarray study. J Cutan Pathol 37: 41-47.

93. Saldanha G, Elshaw S, Sachs P, Alharbi H, Shah P, et al. (2016) microRNA$10 \mathrm{~b}$ is a prognostic biomarker for melanoma. Mod Pathol 29: 112-121.

94. Sengupta D, Tackett AJ (2016) Proteomic Findings in Melanoma. J Proteomics Bioinform 9. 